\title{
Comparison of Clinical Outcomes in Patients With Localized or Locally Advanced Urothelial Carcinoma Treated With Neoadjuvant Chemotherapy Involving Gemcitabine-Cisplatin and High Dose-Intensity MVAC
}

\section{Yongjune Lee}

Asan Medical Center

Young Seok Kim

Asan Medical Center

\section{Bumsik Hong}

Asan Medical Center

Yong Mee Cho

Asan Medical Center

Jae-Lyun Lee ( $\boldsymbol{\nabla}$ jaelyun@amc.seoul.kr)

Asan Medical Center, University of Ulsan College of Medicine https://orcid.org/0000-0002-9420-7162

Original Article - Clinical Oncology

Keywords: Bladder cancer, upper tract urothelial cancer, neoadjuvant chemotherapy, high dose-intensity chemotherapy

Posted Date: February 1st, 2021

DOl: https://doi.org/10.21203/rs.3.rs-164451/v1

License: (c) (i) This work is licensed under a Creative Commons Attribution 4.0 International License. Read Full License

Version of Record: A version of this preprint was published at Journal of Cancer Research and Clinical Oncology on March 14th, 2021. See the published version at https://doi.org/10.1007/s00432-021-03582$x$. 
Comparison of clinical outcomes in patients with localized or locally advanced urothelial carcinoma treated with neoadjuvant chemotherapy involving gemcitabine-cisplatin and high dose-intensity MVAC

Yongjune Lee ${ }^{\mathrm{a}}$, Young Seok Kim ${ }^{\mathrm{b}}$, Bumsik Hong ${ }^{\mathrm{c}}$, Yong Mee Cho ${ }^{\mathrm{d}}$, Jae-Lyun Lee

Departments of ${ }^{\mathrm{a} O n c o l o g y},{ }^{\mathrm{b}}$ Radiation Oncology, ${ }^{\mathrm{c}}$ Urology, and ${ }^{\mathrm{d}}$ Pathology, Asan Medical Center, University of Ulsan College of Medicine, Seoul, Korea

Correspondence: Jae-Lyun Lee, MD

Department of Oncology, Asan Medical Center, University of Ulsan College of Medicine, 88, Olympic-ro 43gil, Songpa-gu, Seoul 05505, Korea

Tel: 82-2-3010-5977

Fax: 82-2-3010-6961

E-mail: jaelyun@amc.seoul.kr

Word count: 2641 words 


\section{Abstract}

Purpose. To compare the efficacy and safety of high dose-intensity combination of methotrexate, vinblastine, adriamycin and cisplatin (HD MVAC) with gemcitabine plus cisplatin (GC) as a neoadjuvant chemotherapy (NAC) in muscle-invasive bladder cancer (MIBC) or locally advanced upper tract urothelial cancer (UTUC). Patients and Methods. A retrospective analysis was conducted for patients with UC (cT2-4aN0-1M0) who received NAC from January 2011 and December 2017 at Asan Medical Center. Pathologic complete response (pCR), down-staging (<ypT2 and no N upstaging), disease-free survival (DFS), OS and safety were compared for each regimen.

Results. Out of a total of 277 patients, 176 patients received GC and 41 patients received HD MVAC. With the exception of age (patients receiving GC were younger; $\mathrm{p}=0.002$ ), other baseline characteristics were well

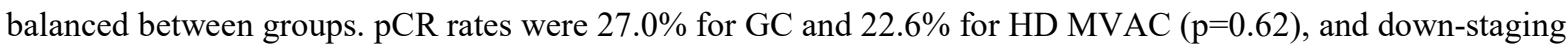
rate was $50.8 \%$ for GC and $58.1 \%$ for HD MVAC ( $\mathrm{p}=0.47)$. There were no differences in OS $(72.1 \%$ vs $73.1 \%$ for GC vs HD MVAC; $\mathrm{p}=0.58)$ and DFS (54.9\% vs 63.3\% for GC vs HD MVAC; $\mathrm{p}=0.21)$ at 3 years. HD MVAC with prophylactic G-CSF was associated with a higher incidence of febrile neutropenia $(p<0.001)$ than GC. The NAC regimen was not an independent prognostic factor for OS.

Conclusions. Oncologic outcomes were not significantly different between the GC and HD MVAC when used as NAC in MIBC/UTUC.

\section{Keywords:}

Bladder cancer; upper tract urothelial cancer; neoadjuvant chemotherapy; high dose-intensity chemotherapy 


\section{Introduction}

It is estimated that 550,000 new cases of bladder cancer occurred worldwide in 2018, with approximately onethird of patients presenting with the muscle-invasive form of the disease (MIBC). More than 20\% of patients with non-muscle invasive bladder cancer progress to MIBC, resulting in 200,000 deaths annually(Bray et al. 2018).

Neoadjuvant chemotherapy (NAC) in MIBC has been established as a standard treatment after SWOG prospective randomized trials demonstrated the efficacy of MVAC as NAC(Grossman et al. 2003). The subsequent meta-analysis of 11 trials encompassing 3,005 patients supported the result that NAC led to an absolute improvement of 5-year overall survival (OS) by 5\% and disease-free survival (DFS) by 9\%(Advanced Bladder Cancer Meta-analysis Collaboration 2003). Despite a high level of evidence, NAC has not been used widely in clinical practice owing to concerns regarding treatment-related toxicity, over-treatment associated with limited accuracy of preoperative staging, and delay to surgery.

In advanced urothelial cancer, the GC (gemcitabine and cisplatin) regimen is preferred to MVAC based on comparative efficacy with a better safety profile and tolerability (von der Maase et al. 2000). Besides, high-dose intensity (HD) MVAC with granulocyte colony-stimulating factor (G-CSF) support had statistically significant survival benefit and higher response rate, especially complete response, than MVAC, with comparable tolerance and fewer dose delays in metastatic urothelial cancer (Sternberg et al. 2006; Sternberg et al. 2001).

Based on data in the metastatic setting, GC or HD MVAC is preferred to MVAC as NAC treatment. Comparative data between GC and HD MVAC in a neoadjuvant setting are limited, and it has not been assessed in randomized controlled trials. The National Comprehensive Cancer Network guideline recommends both GC and HD MVAC as preferred regimens without preference between them(National Comprehensive Cancer Network 2020). In comparison, the European Association of Urology and the American Urologic Association do not suggest specific regimens and refer to cisplatin-based chemotherapy(Alfred Witjes et al. 2017; Chang et al. 2017).

A few retrospective studies recently compared the clinical outcomes of the HD MVAC regimen and the GC regimen when used as NAC in MIBC, which have shown somewhat contradictory results(Peyton et al. 2018; van de Putte et al. 2016; Zargar et al. 2018).

The evidence of NAC in upper tract urothelial cancer (UTUC) is scarce. The POUT study showed adjuvant platinum-based chemotherapy improved disease-free and metastasis-free survival in UTUC(Birtle et al. 2020). However, due to reduced kidney function after nephroureterectomy, the neoadjuvant setting may be preferred over adjuvant for chemotherapy administration in UTUC, especially for a clinical node-positive disease where the chance of over-treatment is minimal(Chakiryan et al. 2019), and retrospective studies have suggested 
survival benefit with NAC(Leow et al. 2014). Similar to bladder cancer, the data of comparative efficacy of NAC regimen in UTUC is lacking.

The aim of this study was therefore to compare the clinical outcomes of neoadjuvant GC with those of HD MVAC in patients with muscle-invasive urothelial bladder cancer.

\section{Patients and Methods}

\section{Patients}

Between January 2011 and December 2017, 290 consecutive patients with urothelial carcinoma who received neoadjuvant chemotherapy at Asan Medical Center, Seoul, Republic of Korea, were reviewed. Eleven patients who had distant metastasis (include M1a) or non-muscle invasive bladder cancer were excluded. All remaining patients were histologically confirmed and documented to have stage cT2-4 N0 M0 or cTany N1 M0 cancer. Sixty-two patients who received NAC other than GC or HD MVAC were excluded. Overall, 217 patients were included in this analysis. The Institutional Review Board of Asan Medical Center approved this study.

\section{Treatment and evaluation}

GC chemotherapy was performed using the following schedule: gemcitabine $1000 \mathrm{mg} / \mathrm{m}^{2}$ on Days 1 and 8 and cisplatin $70 \mathrm{mg} / \mathrm{m}^{2}$ on Day 1, every 3 weeks. HD MVAC chemotherapy involved methotrexate $30 \mathrm{mg} / \mathrm{m}^{2}$ on Day 1, vinblastine $3 \mathrm{mg} / \mathrm{m}^{2}$ on Day 2, doxorubicin $30 \mathrm{mg} / \mathrm{m}^{2}$ and cisplatin $70 \mathrm{mg} / \mathrm{m}^{2}$ on Day 2, and G-CSF 300 $\mu \mathrm{g} / \mathrm{m}^{2}$ from Days 4-10 or long-acting G-CSF(pegfilgrastim) on Day 2, every 2 weeks. In both groups, patients without $\mathrm{cN} 1$ received four cycles of chemotherapy, whereas those with $\mathrm{cN} 1$ received six cycles if there was no evidence of disease progression and adverse events were tolerable.

Surgery was conducted only if all lesions were resectable, assessed by the urologic surgeon after NAC. Patients received partial or radical cystectomy, radical nephroureterectomy, or segmental ureterectomy, according to the lesions involved. No surgery was performed in case of clinical disease progression(cPD) to NAC. In the case of medically inoperable patients or refusal of surgery, concurrent chemoradiation or radiotherapy was recommended after NAC. Repeated CT scans were obtained immediately after neoadjuvant chemotherapy before surgery for $\mathrm{cN} 0$ disease, while we did additional CT scans after the 3rd cycle of NAC for patients with $\mathrm{cN} 1$ disease.

We defined the extent of resection as being macroscopically complete with a negative microscopic margin (R0), macroscopically complete with a positive microscopic margin (R1), or macroscopically incomplete (R2). We determined the pathological response based on cystectomy and pelvic lymph node dissection (PLND) for patients who underwent surgery. PLND was performed per a standardized template. We reviewed the rate of the patient's pathologic down-staging and pathologic complete response (pCR). Down-staging was defined as $<y p T 2$ and no $\mathrm{N}$ upstaging at operation. pCR was defined as no evidence of residual tumor (ypT0N0). Toxicity 
during chemotherapy was classified in accordance with the Common Terminology Criteria for Adverse Events (CTCAE) v4.03(National Cancer Institute 2010).

\section{Statistical analysis}

OS was defined as the duration of time from the start date of NAC to the date of death due to any cause. DFS was defined as the duration of time from the start date of neoadjuvant chemotherapy starting to the date of disease recurrence or death due to any cause, whichever occurred first. Survival rates and corresponding standard errors were estimated using the Kaplan-Meier method, and survival curves were compared using the log-rank test. Baseline characteristics, clinical response rates, pathologic down-staging rate, $\mathrm{pCR}$ between groups were compared using Pearson's chi-square test or Fisher's exact test for categorical variables and Student's $t$-test or Mann Whitney U test for continuous variables, as appropriate. To identify clinical prognostic factors for OS and DFS, univariate and multivariate analyses were performed using Cox proportional hazard regression modeling. The key baseline characteristics and candidate prognostic factors, including age, sex, tumor histology, clinical stage, hydronephrosis at presentation, history of non-muscle invasive bladder cancer, and neoadjuvant regimen were included in the univariate analysis. Variables exhibiting a potential association with survival $(P<0.25)$ in the univariate analysis and neoadjuvant regimen were included in the multivariate analysis. All analyses were computed using SPSS Statistics version 24 (IBM SPSS Inc. Armonk, NY, USA). All tests were two-sided, with $P$ values of $<0.05$ considered statistically significant.

\section{Results}

\section{Patient characteristics}

The baseline characteristics of patients in the GC $(n=176)$ and HD MVAC $(n=41)$ groups are presented in Table 1. The characteristics did not differ significantly between the two groups, with the exception of age: patients treated with HD MVAC were younger than those treated with $\mathrm{GC}(p=0.002)$.

\section{Neoadjuvant chemotherapy, administration, clinical response, and tolerability}

The number of median chemotherapy cycles was 4 (IQR 3-4) for the GC group and 4 (IQR 3-5.5) for the HD MVAC group. All patients received at least two cycles of neoadjuvant chemotherapy. The percentage of patients who received fewer than 3 cycles of chemotherapy was $10.2 \%$ in the GC group and $2.4 \%$ in the HD MVAC group ( $\mathrm{p}=0.135$ ). The clinical responses to NAC for both groups are listed in Supplement Table 1. There were no differences in clinical response rate between the groups: the cCR rate was $28.4 \%$ in the $\mathrm{GC}$ group and $17.1 \%$ in the HD MVAC group, and the cPD rate was $4.5 \%$ in GC group and $7.3 \%$ in HD MVAC group ( $\mathrm{p}=0.337)$. Grade 3 or worse hematologic adverse events that occurred are presented in Table 2. The incidence of CTCAE Grade $3 / 4$ neutropenia was $46.6 \%$ in the GC group and $19.5 \%$ in the HD MVAC group; these values are significantly different $(\mathrm{p}=0.002)$. Despite the higher incidence of Grade $3 / 4$ neutropenia in the GC group, the HD MVAC with prophylactic G-CSF group was associated with a higher incidence of febrile neutropenia than GC $(0.6 \%$ in the GC group vs. $12.2 \%$ in the HD MVAC group, $\mathrm{p}<0.001)$. The incidences of severe anemia (5.7\% in the GC group vs. $9.8 \%$ in the HD MVAC group, $\mathrm{p}=0.308)$ and thrombocytopenia $(10.2 \%$ in the GC 
group vs. $12.2 \%$ in the HD MVAC group, $\mathrm{p}=0.217$ ) were comparable between the two groups. Severe nonhematologic adverse events are detailed in Supplement 2.

\section{Surgery and pathologic outcomes}

Overall, 71\% of patients underwent surgery after NAC. The proportion of patients who underwent surgery was not different between the two groups (69.3\% in the GC group vs. $75.6 \%$ in the HD MVAC group, $\mathrm{p}=0.426$, Table 3).

The rate of incomplete resection was $9 \%(n=11)$ in the GC group and 13\% $(n=4)$ in the HD MVAC group. The down-staging rate was $50.8 \%$ in the GC group and $58.1 \%$ in the HD MVAC group $(\mathrm{p}=0.470)$. The pCR rate was $27.0 \%$ in the GC group and $22.6 \%$ in the HD MVAC group ( $\mathrm{p}=0.613)$.

\section{Survival outcomes}

The survival outcome of patients with UC is shown by NAC regimen in Figure 1. With a median follow-up duration of 37 months, there were no differences in OS and DFS between groups. The 3-year OS was $72.1 \%$ in the GC group and $73.1 \%$ in the HD MVAC group, the 5-year OS was $63.8 \%$ in the GC group and $67.9 \%$ in the HD MVAC group $(\mathrm{HR}=1.21 ; 95 \% \mathrm{CI}, 0.60-2.43 ; \mathrm{p}=0.588)$, the 3 -year DFS was $54.9 \%$ in the GC group and $63.2 \%$ in the HD MVAC group, and the 5-year DFS was $43.5 \%$ in the GC group and $63.2 \%$ in the HD MVAC group $(\mathrm{HR}=1.42 ; 95 \% \mathrm{CI}, 0.81-2.49 ; \mathrm{p}=0.211)$.

\section{Subgroup analysis}

Our study consisted of patients with bladder cancer, upper tract urothelial cancer (UTUC), and both site involvement. We analyzed two groups of patients, bladder only subgroup and the UTUC subgroup (patients with upper tract lesions); the subgroups contained 180 and 37 patients, respectively.

The baseline characteristics of the bladder subgroup were not significantly difference, except in terms of age (Supplement Table 3). Neither the clinical outcomes (Supplement Table 4) nor the pathologic outcomes differed significantly between the two regimens (Supplement Table 5). The down-staging rate of the bladder-involved subgroup was $55.3 \%$ for GC and 55.6\% for HD MVAC ( $\mathrm{p}=0.983)$, and the pCR rate was $31.9 \%$ for GC and $22.2 \%$ for HD MVAC $(\mathrm{p}=0.510)$. In the UTUC subgroup, there were no significant differences in pathologic outcomes (Supplement Table 6). But a numerically higher proportion of patients treated with HD MVAC achieved pCR (25\% vs. $10.7 \%)$ and down-staging (75\% vs. $35.7 \%)$.

\section{Prognostic factors affecting survival outcomes}

The univariate and multivariate analyses of the potential prognostic factors for DFS and OS are summarized in Table 4. In the univariate analysis, the TNM stage, hydronephrosis, anemia, and down-staging to NAC were statistically significant factors associated with OS. Among them down-staging alone remained as significant factor affecting OS and DFS in multivariate analysis. NAC was not a statistically significant prognostic factor for neither OS nor DFS. 


\section{Discussion}

Our study showed that the HD MVAC regimen when used as NAC did not show superiority in efficacy and safety in patients with MIBC/UTUC compared with the GC regimen. There were no statistically significant differences in pCR, down-staging rate, OS, and DFS between groups. The proportion of patients who were not operated on owing to clinical progression or a deteriorated condition associated with adverse events was comparable between groups. Even though prophylactic G-CSF was given to all patients receiving HD MVAC, febrile neutropenia occurred more frequently in patients treated with HD MVAC group.

There are only four published studies that compared GC and HD MVAC regimens used as NAC for bladder cancer (Table 5). Three of the studies were retrospective observational analysis, and one study was a prospective randomized phase II trial with a primary endpoint of regimen-specific COXEN score (Flaig et al. 2019; Peyton et al. 2018; van de Putte et al. 2016; Zargar et al. 2018). In contrast to our study, Peyton et al. and Zargar et al. showed that the HD MVAC regimen led to higher pCR and longer OS than the GC regimen(Peyton et al. 2018; Zargar et al. 2018). Similar to our analysis, Van de Putte et al. showed no differences in pCR rates between GC and HD MVAC(van de Putte et al. 2016). In the SWOG S1314 trial (COXEN Trial), the comparison of the efficacy between the two regimens was not the primary objective, but this is the only prospective study published to have shown no difference in pCR and pPR $(<\mathrm{pT} 2)$ between the two regimens(van de Putte et al. 2016). The results of our analysis were consistent with those of this prospective trial. Given the fact that the proportions of patients with incomplete NAC cycles $(<3)$ and those who did not undergo surgery were higher in the GC group in the current study, no statistical difference in pCR rate and OS suggests that it would be difficult to achieve better clinical outcome with just change of regimen from GC to HD MVAC.

The pCR rate in the HD MVAC group was $27.0 \%$ and $22.7 \%$ for GC group in the current study. Peyton et al. and the SWOG S1314 (COXEN) study showed a higher pCR rate than others(Flaig et al. 2019; Peyton et al. 2018). The reason for this is probably higher proportions of patients with cT2 disease in these studies, $68.7 \%$ and $87 \%$, respectively. The pCR rate in the HD MVAC group in the current study was comparable with that reported by Choueiri et al., which enrolled patients with similar baseline clinical characteristics with the current study(Choueiri et al. 2014).

Although Grade 3 or higher neutropenia was more frequent in the GC group, the incidence of febrile neutropenia was significantly higher in the HD MVAC group, which were in line with the study reported by Van de Putte et al.(van de Putte et al. 2016) The majority of neutropenia encountered in the GC group was found on laboratory exam on day 8. It did not lead to clinically significant neutropenic fever if dose reduction or dose delay of gemcitabine is adequately employed. Besides, grade 3 or worse mucositis developed only in patients in the HD MVAC group. From the perspective of adverse events, the GC regimen seems better and more tolerable.

The present study has several limitations. As anticipated from retrospective study design, selection bias may have occurred. Indeed, there was a statistically significant difference in the age between the HD MVAC and GC groups. The inclusion of patients with UTUC may have influenced the results of the analysis. However, in subgroup 
analysis involving only patients with MIBC, there were no significant differences in clinical and pathological outcomes. The difference in cohort size between the two groups and the small sample size in total may also have reduced the statistical power to assess the benefit of HD MVAC. A possible underestimation of toxicity may also have occurred because of different sampling points. Also, patients' co-morbidity and performance status data were not captured and may act as a confounding factor for the statistical analysis.

The ongoing clinical trial results, the GETUG/AFU V05 VESPER trial, are eagerly needed to draw a firm conclusion on the benefit of HD MVAC over GC. However, as the VESPER trial compares six cycles of HD MVAC with four cycles of GC, a new question on an adequate number of NAC would be incurred.

\section{Conclusions}

Our findings did not show the superiority of the neoadjuvant HD MVAC regimen to the GC regimen in terms of efficacy and safety in patients with localized or locally advanced urothelial cancer.

\section{Acknowledgments}

Funding Nothing to be declared

Conflict of Interest The authors declare that there are no relevant conflict of interests.

Ethics approval IRB of Asan Medical Center reviewed and approved this study.

Consent for publication All authors consent to publish this study in J Cancer Res Clin Oncol.

\section{Author contributions:}

Dr YJ Lee and JL Lee had full access to all the data in the study and takes responsibility for the integrity of the data and the accuracy of the data analysis.

Concept and design: YJ Lee, JL Lee

Acquisition, analysis, or interpretation of data: YJ Lee, JL Lee, YS Kim, BS Hong, YM Cho, JL Lee

Drafting of the manuscript: YJ Lee, JL Lee

Critical revision of the manuscript for important intellectual content: YJ Lee, JL Lee, YS Kim, BS Hong, YM

Cho, JL Lee

Statistical analysis: YJ Lee, JL Lee

Administrative, technical, or material support: YJ Lee, JL Lee, YS Kim, BS Hong, YM Cho, JL Lee

Data analysis: YJ Lee, JL Lee

\section{References}

Advanced Bladder Cancer Meta-analysis Collaboration (2003) Neoadjuvant chemotherapy in invasive bladder cancer: a systematic review and meta-analysis Lancet 361:1927-1934 doi:10.1016/s0140$6736(03) 13580-5$

Alfred Witjes J et al. (2017) Updated 2016 EAU Guidelines on Muscle-invasive and Metastatic Bladder Cancer 
Eur Urol 71:462-475 doi:10.1016/j.eururo.2016.06.020

Birtle A et al. (2020) Adjuvant chemotherapy in upper tract urothelial carcinoma (the POUT trial): a phase 3, open-label, randomised controlled trial Lancet 395:1268-1277 doi:10.1016/S0140-6736(20)30415-3

Bray F, Ferlay J, Soerjomataram I, Siegel RL, Torre LA, Jemal A (2018) Global cancer statistics 2018: GLOBOCAN estimates of incidence and mortality worldwide for 36 cancers in 185 countries CA Cancer J Clin 68:394-424 doi:10.3322/caac.21492

Chakiryan N, Martinez A, Gao L, Liu JJ, Amling C, Garzotto M, Kopp RP (2019) Optimizing the Sequence of Chemotherapy for Upper Tract Urothelial Carcinoma with Clinically Positive Regional Lymph Nodes J Urol 202:76-82 doi:10.1097/JU.0000000000000172

Chang SS et al. (2017) Treatment of Non-Metastatic Muscle-Invasive Bladder Cancer: AUA/ASCO/ASTRO/SUO Guideline J Urol 198:552-559 doi:10.1016/j.juro.2017.04.086

Choueiri TK et al. (2014) Neoadjuvant dose-dense methotrexate, vinblastine, doxorubicin, and cisplatin with pegfilgrastim support in muscle-invasive urothelial cancer: pathologic, radiologic, and biomarker correlates J Clin Oncol 32:1889-1894 doi:10.1200/jco.2013.52.4785

Flaig TW et al. (2019) SWOG S1314: A randomized phase II study of co-expression extrapolation (COXEN) with neoadjuvant chemotherapy for localized, muscle-invasive bladder cancer Journal of Clinical Oncology 37:4506-4506 doi:10.1200/JCO.2019.37.15_suppl.4506

Grossman HB et al. (2003) Neoadjuvant chemotherapy plus cystectomy compared with cystectomy alone for locally advanced bladder cancer N Engl J Med 349:859-866 doi:10.1056/NEJMoa022148

Leow JJ, Martin-Doyle W, Fay AP, Choueiri TK, Chang SL, Bellmunt J (2014) A systematic review and metaanalysis of adjuvant and neoadjuvant chemotherapy for upper tract urothelial carcinoma Eur Urol 66:529-541 doi:10.1016/j.eururo.2014.03.003

National Cancer Institute (2010) Common Terminology Criteria for Adverse Events (CTCAE):Version 4.03. https://ctep.cancer.gov/protocoldevelopment/electronic_applications/docs/CTCAE_4.03.xlsx. Accessed July 1, 20202020

National Comprehensive Cancer Network (2020) Bladder Cancer (version 6.2020). https://www.nccn.org/professionals/physician_gls/pdf/bladder.pdf. Accessed January 15, 20212021

Peyton CC et al. (2018) Downstaging and Survival Outcomes Associated With Neoadjuvant Chemotherapy Regimens Among Patients Treated With Cystectomy for Muscle-Invasive Bladder Cancer JAMA Oncol 4:1535-1542 doi:10.1001/jamaoncol.2018.3542

Sternberg CN et al. (2006) Seven year update of an EORTC phase III trial of high-dose intensity M-VAC chemotherapy and G-CSF versus classic M-VAC in advanced urothelial tract tumours Eur J Cancer 42:50-54 doi:10.1016/j.ejca.2005.08.032

Sternberg CN et al. (2001) Randomized phase III trial of high-dose-intensity methotrexate, vinblastine, doxorubicin, and cisplatin (MVAC) chemotherapy and recombinant human granulocyte colonystimulating factor versus classic MVAC in advanced urothelial tract tumors: European Organization for Research and Treatment of Cancer Protocol no. 30924 J Clin Oncol 19:2638-2646 doi:10.1200/jco.2001.19.10.2638

van de Putte EE et al. (2016) Neoadjuvant induction dose-dense MVAC for muscle invasive bladder cancer: 
efficacy and safety compared with classic MVAC and gemcitabine/cisplatin World J Urol 34:157-162 doi:10.1007/s00345-015-1636-y

von der Maase H et al. (2000) Gemcitabine and cisplatin versus methotrexate, vinblastine, doxorubicin, and cisplatin in advanced or metastatic bladder cancer: results of a large, randomized, multinational, multicenter, phase III study J Clin Oncol 18:3068-3077

Zargar H et al. (2018) Neoadjuvant Dose Dense MVAC versus Gemcitabine and Cisplatin in Patients with cT34aN0M0 Bladder Cancer Treated with Radical Cystectomy J Urol 199:1452-1458 doi:10.1016/j.juro.2017.12.062 
Figure legends

Figure 1. Survival outcomes according to NAC regimen 
Figures
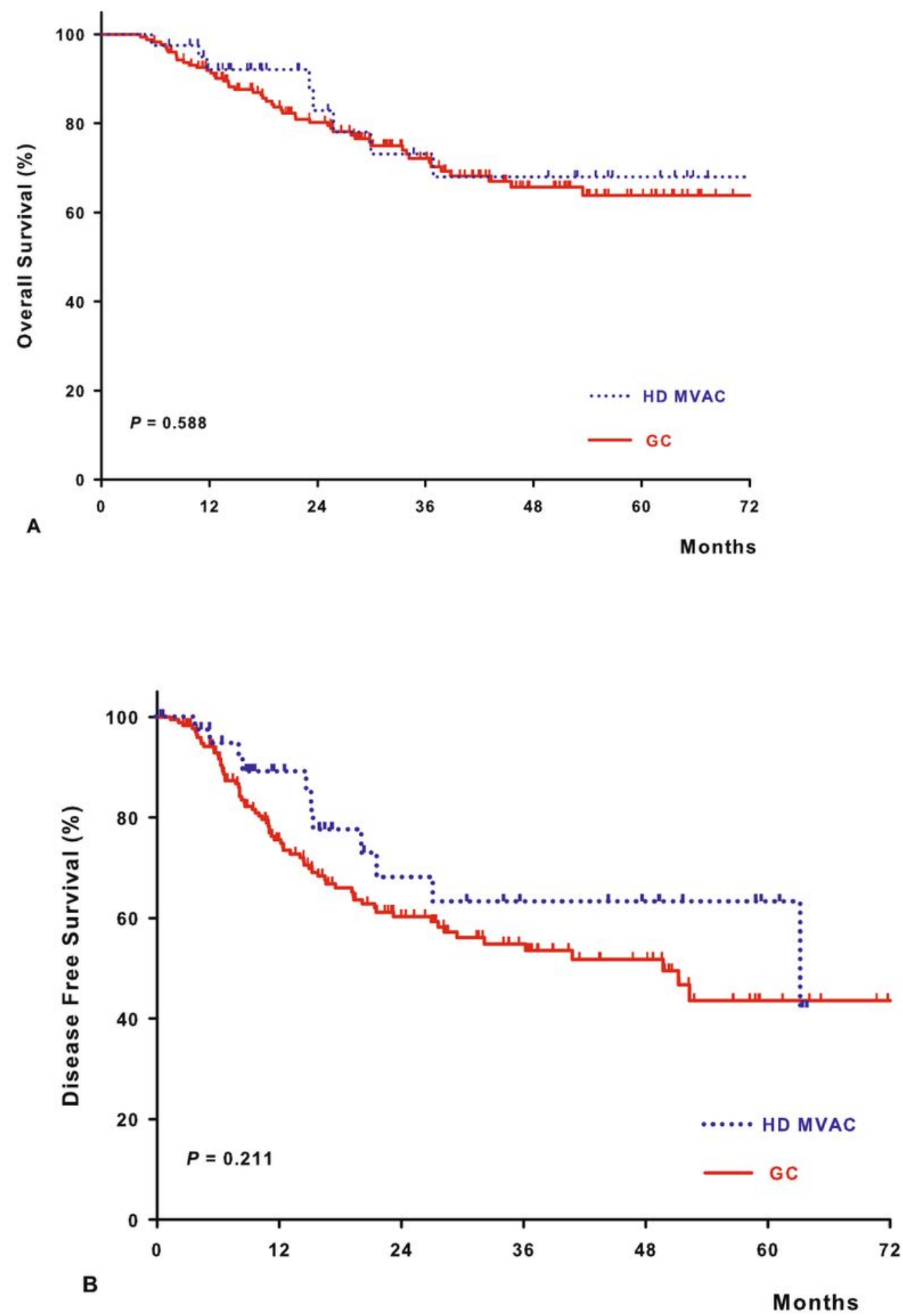

Figure 1

Survival outcomes according to NAC regimen

\section{Supplementary Files}


This is a list of supplementary files associated with this preprint. Click to download.

- TablesOnly.pdf 